\title{
COMPREHENSIVE TREATMENT FOR TIC DISORDERS
}

\author{
Omar Rahman, \& Adam Lewin \\ University of South Florida (USA)
}

\begin{abstract}
Purpose: This workshop will help mental health professionals increase knowledge of tic disorders.

Background: Tic disorders, including Tourette's, affect around 1 in 100 children, with many continuing to have tics in adulthood. Impairment can be in the areas of emotional difficulties, social problems, family problems, school/work, physical problems, sleep issues, etc. Often, the nature of tics is unpredictable and uncontrollable, which leads to an increased sense of frustration.

Tic disorders are often comorbid with other conditions, such as OCD, ADHD, depression, and anxiety. In addition, many individuals with Tourette's have difficulty with emotional control, outbursts, and rage. Often, these are misunderstood by others and addressed in unhelpful ways.

Knowledge about the nature of tics, common issues, and treatment strategies, is typically low, even in the mental health field. Additionally, tics can sometimes be difficult to identify, or may be confused with other movements or sounds. Attendees of this workshop will better be able to identify tics, common problems, and successful treatment strategies.

Key points: We will discuss:

-Differential identification of tics (vs. compulsions, movements, stereotypies)

-Comprehensive Behavioral Interventions for Tics (CBIT), including Habit Reversal Training

-Pharmacological treatment

-Managing typical comorbid conditions and reducing dysfunction

-Ongoing research

Procedure:

-Information will be presented by an expert in a discussion format, with interaction and questions from attendees. Short videos will be used for demonstrating common tics and interventions. There will be interactive demonstration of some of the major techniques.

Description of the participants: Participants will be mental health professionals or others who wish to develop knowledge in identification and treatment of tic disorders. Maximum number will be 40 .
\end{abstract}

Keywords: Tics, Tourette's, CBIT, HRT. 